\title{
Genetic modifiers of sickle cell anaemia in patients from Nigeria: A pilot study
}

Obiageli Nnodu', Hezekiah Isa', Yohanna Tanko', Theresa Otu ${ }^{1}$, Jacqueline Ekong ${ }^{3}$, Zakaria Elmi², Helen Rooks², Stephan Menzel², Rosemary Ekong ${ }^{3}$

${ }^{1}$ Centre of Excellence for Sickle Cell Disease Research and Training, University of Abuja (CESRTA), Nigeria.

${ }^{2}$ Comprehensive Cancer Centre, King's College London, UK.

${ }^{3}$ Department of Genetics, Evolution and Environment, University College London, UK.

The variable clinical phenotype in sickle cell anaemia (SCA) is well documented, with severity in presentation partly associated with the persistence of fetal haemoglobin ( $\mathrm{HbF})$ and co-inheritance of $\alpha$-thalassemia ( $\alpha-3.7$ globin gene deletion). Understanding genetic factors that contribute to clinical variability and severity in SCA is one step towards tackling health issues in SCA, as well as advancing knowledge about the disease, and research geared towards novel therapeutic targets. Genetic modifiers of $\mathrm{HbF}$ have been identified in several global populations, with a few studies reported from Nigeria.

We conducted a pilot study in the Federal Capital Territory of Nigeria to: (1) survey the prevalence of known genetic modifiers of $\mathrm{HbF}$ and the co-inheritance of $\alpha$-thalassemia in a different region of Nigeria; (2) boost capacity for sickle cell disease (SCD) research in Nigeria through collaborative studies; and (3) troubleshoot procedures for the investigation.

Our findings indicate: (a) differences in the frequencies of HbF-boosting alleles between the Abuja and Lagos patient groups; (b) associations between HbF levels and, HMIP-2A (rs66650371) and Xmn1-HBG2 (rs7482144); (c) HbF-boosting alleles observed in cis within $B C L 11 A$ in the Abuja study; (d) negative correlation between $3.7 \mathrm{~kb}$ alpha globin gene deletion and, MCV and $\mathrm{MCH}$; and (e) no major administrative or operational obstacles in this collaborative study. This study presents the basis for a larger and regional SCD study to aid the management of patients with SCD, and the development and implementation of relevant healthcare policies incorporating the genomics of SCD in Nigeria.

Key words: Sickle cell anaemia, genetic modifiers, co-inheritance of alpha-thalassemia, Nigeria

Funding: TET Fund, UCL Global Engagement Fund, Wellcome Trust 211923/Z/18/Z 